\title{
Partial Rescue of Retinal Function and Sterol Steady-State in a Rat Model of Smith-Lemli-Opitz Syndrome
}

\author{
STEVEN J. FLIESLER, DANA K. VAUGHAN, ERIN C. JENEWEIN, MICHAEL J. RICHARDS, BARBARA A. NAGEL, \\ AND NEAL S. PEACHEY
}

\begin{abstract}
Departments of Ophthalmology and Pharmacological and Physiological Science [S.J.F., M.J.R., B.A.N.], Saint Louis University School of Medicine, St. Louis, Missouri, 63104; Department of Biology [D.K.V., E.C.J.], University of Wisconsin Oshkosh, Oshkosh,

Wisconsin, 54901; Cleveland Veterans Association Medical Center and Cole Eye Institute [N.S.P.], The Cleveland Clinic Foundation, Cleveland, Ohio, 44195
\end{abstract}

\begin{abstract}
The Smith-Lemli-Opitz syndrome (SLOS) is the firstdescribed in a growing family of hereditary defects in cholesterol biosynthesis, and presents with a spectrum of serious abnormalities, including multiple dysmorphologies, failure to thrive, cognitive and behavioral impairments, and retinopathy. Using a pharmacologically induced rat model of SLOS that exhibits key hallmarks of the disease, including progressive retinal degeneration and dysfunction, we show that a high-cholesterol diet can substantially correct abnormalities in retinal sterol composition, with concomitant improvement of visual function, particularly within the cone pathway. Although histologic degeneration still occurred, a high-cholesterol diet reduced the number of pyknotic photoreceptor nuclei, relative to animals on a cholesterol-free diet. These findings demonstrate that cholesterol readily crosses the blood-retina barrier (unlike the blood-brain barrier) and suggest that cholesterol supplementation may be efficacious in treating SLOS-associated retinopathy. (Pediatr Res 61: 273-278, 2007)
\end{abstract}

$\mathrm{H}$ ereditary abnormalities in cholesterol biosynthesis underlie a family of human birth defects associated with specific enzyme defects and phenotypes (1-3). The first described and most common of these is SLOS (4), a multiple congenital anomalies syndrome involving the inability to efficiently convert $7 \mathrm{DHC}$ to $\mathrm{Chol}(5,6)$. The enzyme responsible for catalyzing this biosynthetic step ( $3 \beta$-hydroxysterol- $\Delta^{7}$ reductase; DHCR7; EC 1.3.1.21) is encoded by the DHCR7 gene localized to human chromosome 11q12-q13 (7-9). More than 100 mutations in DHCR7 have been identified in association with SLOS; many result in abnormally low levels of cholesterol and abnormally high levels of 7DHC in bodily tissues $(8,9)$. With an estimated global incidence of 1 in 20,000 to 1 in 60,000 live births, SLOS is likely the fourth most common human recessive disease, after cystic fibrosis, phenylketonuria, and hemochromatosis $(8,9)$. However, a recent study (10) estimates the SLOS carrier frequency to be 1 in 30, suggesting a much higher actual disease frequency, e.g. approximately 1 in 1,590 to 1 in 13,500. The SLOS phenotype

Received August 22, 2006; accepted October 19, 2006.

Correspondence: Steven J. Fliesler, Ph.D., Saint Louis University Eye Institute, 1755 S. Grand Blvd., St. Louis, MO 63104-1540; e-mail: Fliesler@slu.edu

Supported by grants from the U.S. Public Health Service [R01EY007361 (SJF), R24EY15638 (NSP)]; March of Dimes grant FY-01-339 (SJF); Research to Prevent Blindness (SJF, NSP); the University of Wisconsin-Oshkosh Faculty Development Program and Graduate School (DKV); and the Department of Veteran Affairs (NSP).

DOI: $10.1203 /$ pdr.0b013e318030d1cf displays a spectrum of clinical manifestations, from mild to lethal, and may include such findings as moderate to severe mental retardation, autism, dysmorphic craniofacial and skeletal malformations, and failure to thrive (1-3).

We previously reported progressive retinal degeneration in a pharmacological rat model of SLOS (11) characterized by shortening and eventual loss of retinal ROS, pyknosis and thinning of the ONL, accumulation of membranous inclusions and lipid droplets in the retinal pigment epithelium (RPE), defects in rod and cone photoresponses, together with SLOSlike accumulation of 7DHC and diminished cholesterol levels in retina, brain, liver, and serum. The SLOS rat model (11-13) is induced by treatment of pregnant rats and their progeny with AY9944, a selective inhibitor of $3 \beta$-hydroxysterol- $\Delta^{7}$ reductase $(14,15)$, the enzyme defective in SLOS. The clinical relevance of the SLOS rat model is highlighted by the fact that pediatric SLOS patients exhibit markedly abnormal darkadapted (rod) ERG (16), similar to those observed in the SLOS rat model. As predicted from our rat model, cone ERG defects also have been observed in SLOS patients (Anne Fulton, personal communication). However, an associated retinal degeneration in SLOS has not been thoroughly documented, due to lack of suitable postmortem human ocular specimens. The single published report of retinal histologic and ultrastructural defects associated with SLOS (17), based upon postmortem tissue specimens harvested from a 1-mo-old male child, has features consistent with the SLOS rat model. Taken together, these findings predict that SLOS has an associated retinal degeneration involving biochemical, histologic, and functional abnormalities in rods and cones. Furthermore, constant bright light exposure exacerbates photoreceptor degeneration in the rat SLOS model (18), far more so than in normal, age-matched albino rats, and the severity of the histologic degeneration correlates with the levels of lipid hydroperoxides present in the retina (19).

Exogenous cholesterol administration is the current ameliorative therapy for SLOS patients (20-22). However, while this can effectively raise serum cholesterol levels, correction of

Abbreviations: 7DHC, 7-dehydrocholesterol; cd, candela; Chol, cholesterol; ERG, electroretinogram; ONL, outer nuclear layer; ROS, rod outer segment; SLOS, RSH/Smith-Lemli-Opitz syndrome 
other clinical manifestations appears to be quite variable and limited (23). Whereas most bodily tissues can readily take up cholesterol, the blood-brain barrier interferes with this process, at least after early postnatal life (24). Hence, neurologic and behavior abnormalities associated with SLOS tend to persist even with cholesterol supplementation (23). Until recently, it was not known whether similar limitations applied to the retina, which is part of the CNS. However, based upon experiments performed with the naturally fluorescent cholesterol analog cholesta-5,7,9(11)-trienol, it has been demonstrated that exogenous LDL and, to a lesser extent, HDL, can efficiently deliver sterols to the neural retina in the rat (25). Herein, we demonstrate that dietary Chol supplementation can dramatically alter the steady-state sterol composition of the retina, restoring near-normal Chol levels and reducing 7-DHC levels, with concomitant improvement of retina electrophysiological function and reduced photoreceptor pyknosis.

\section{MATERIALS AND METHODS}

Animals. All procedures were approved by Institutional Animal Care and Use Committees (IACUCs) of Saint Louis University and the Cleveland VAMC and conformed to the National Institutes of Health Guide for the Care and Use of Laboratory Animals. Rats were maintained in dim cyclic light (20-40 lux, 12-h light/12-h dark) at $22-25^{\circ} \mathrm{C}$.

Pregnant Sprague-Dawley rats (Harlan Bioproducts for Science, Indianapolis, IN; $6 \mathrm{~d}$ sperm-positive) were fed Chol-free chow ( $0 \%$ Chol; Purina Mills Test Diet, Richmond, IN) and administered custom-synthesized AY9944 $(0.37 \mathrm{mg} / \mathrm{kg} / \mathrm{d}$, in PBS solution, at $2.5 \mu \mathrm{L} / \mathrm{h})$ via subcutaneous Alzet osmotic pumps (model 2ML4; Durect Corp., Cupertino, CA) from gestational d 7 to weaning. Control dams were fed $0 \%$ Chol chow and given no other treatment. Pups from AY9944-treated dams were injected subcutaneously with AY9944 $(30 \mathrm{mg} / \mathrm{kg}$, in PBS) three times per week on alternating days, starting at postnatal d 1 (P1) and continuing throughout life, as previously described $(11,13)$. These animals also were injected concomitantly with vitamins A, D, and E (Vital-A, D+E; Schering-Plough, Kenilworth, NJ) as described previously $(11,13)$, which dramatically improves postnatal viability (13). Control pups received no vitamin injections, as this did not affect survival, retinal histology or function, or sterol levels in normal rats (unpublished results).

Upon weaning (postnatal d 28), AY9944-treated animals were split into two groups: one (2\% Chol group) was fed a $2 \%$ (by weight) Chol-enriched chow (Purina), whereas the other (0\% Chol group) was fed Chol -free chow. Both groups continued with vitamin injections in parallel, and were maintained on this regimen through postnatal wk 10.

Electroretinography. At $10 \mathrm{wk}$ of age, dark-adapted (rod) and lightadapted (cone) ERG were recorded from anesthetized animals, as described in detail elsewhere (11).
Biochemical analysis. Following ERG measurements, deeply anesthetized animals were killed by withdrawing intracardiac blood by syringe; after clotting (at $4^{\circ} \mathrm{C}$, protected from light), serum was prepared by centrifugation and then frozen in liquid nitrogen. Brains and livers were harvested, quickly rinsed in ice-chilled PBS, blotted dry, and frozen in liquid nitrogen. One retina was harvested and frozen; the contralateral eye was enucleated and fixed with buffered mixed aldehydes for histologic and morphometric analyses (11). All tissues were stored in darkness at $-85^{\circ} \mathrm{C}$ until ready for further processing. Portions of liver, brain, serum, and the entirety of one retina per animal were saponified, extracted with petroleum ether, and analyzed by reverse-phase HPLC, as previously described in detail $(11,13)$.

Histologic and morphometric analysis. The methods used for histologic and quantitative morphometric analysis of retinas were as described in detail previously $(11,18)$.

Statistics. The $t$ test (two-tailed) was applied to the quantitative sterol data using the statistics software provided in Microsoft Excel (Microsoft, Redmond, WA). Statistical analysis of quantitative morphometric data and ERG data used a two-way repeated measures ANOVA. In both cases, a value of $p<0.05$ was considered statistically significant.

\section{RESULTS}

Effect of dietary cholesterol supplementation on steadystate sterol composition. Quantitative sterol composition data for serum, retina, brain, and liver specimens obtained from AY9944-treated rats, plus and minus 2\% dietary cholesterol supplementation, are shown in Table 1. In agreement with our prior studies $(11,13)$, tissues from normal age-matched controls contained essentially no 7DHC; Chol represented $>98 \%$ of total sterols, and feeding a $2 \%$ Chol-enriched diet did not significantly alter the steady-state sterol composition or content (data not shown). In contrast, animals administered AY9944 alone (0\% Chol group) exhibited marked accumulation of 7DHC in all tissues. For example, the 7DHC/Chol mole ratio in serum was $7.3( \pm 1.92 ; n=6)$, and total sterols were dramatically lower than normal $(25 \mathrm{mg} / \mathrm{dL}$, compared with $100-120 \mathrm{mg} / \mathrm{dL}$ in age-matched controls). In retina, this ratio was $5.65( \pm 0.49 ; n=6)$ in the $0 \%$ Chol group. In the $2 \%$ Chol group, the 7DHC/Chol mole ratio of retina plummeted to $1.40( \pm 0.1)$, largely due to the 3 -fold increase in the retina Chol content, rather than the 0.3 -fold reduction in $7 \mathrm{DHC}$ levels. No statistically significant differences in total retina sterol content were observed; hence, this dramatic change in sterol composition reflects a one-for-one molecular replacement of 7DHC with Chol obtained via dietary supplementation.

Table 1. Effect of dietary Chol supplementation on sterol composition of tissues from AY9944-treated rats, relative to AY9944-treated rats fed a Chol-free diet

\begin{tabular}{|c|c|c|c|c|c|c|c|c|}
\hline & \multicolumn{2}{|c|}{ Serum** } & \multicolumn{2}{|c|}{ Liver† } & \multicolumn{2}{|c|}{ Retina $\ddagger$} & \multicolumn{2}{|c|}{ Brain $\dagger$} \\
\hline 7DHC/Chol*,§ & $7.30 \pm 1.92$ & $0.10 \pm 0.00$ & $5.52 \pm 2.68$ & $0.03 \pm 0.01$ & $5.65 \pm 0.49$ & $1.40 \pm 0.10$ & $2.95 \pm 1.78$ & $1.95 \pm 1.35$ \\
\hline Chol§ & $3.27 \pm 1.51$ & $105.2 \pm 14.9$ & $0.33 \pm 0.29$ & $8.18 \pm 3.76$ & $8.61 \pm 0.97$ & $23.6 \pm 2.90$ & $2.78 \pm 1.68$ & $3.27 \pm 1.23$ \\
\hline Total\| & $25.5 \pm 9.13$ & $115.7 \pm 16.4$ & $1.73 \pm 0.61$ & $8.43 \pm 3.76$ & $57.2 \pm 3.30$ & $56.0 \pm 6.40$ & $9.05 \pm 3.42$ & $8.47 \pm 1.92$ \\
\hline
\end{tabular}

Sprague-Dawley rats were treated throughout their lifetime, from gestational d 6 through postnatal (PN) d 74, with AY9944, with diets from PN28 to PN 74. Quantification of Chol and 7DHC determined by reverse-phase HPLC of nonsaponifiable lipid extracts, in comparison with authentic sterol standards (values are mean $\pm \mathrm{SD}, n=6)$.

* For all tissues, 7DHC/Chol expressed as mole ratio.

** Values expressed as $\mathrm{mg} / \mathrm{dL}$.

$\dagger$ Values expressed as $\mathrm{mg} / \mathrm{g}$ wet weight.

$\ddagger$ Values expressed as nmol/retina.

$\S$ Values for all tissues except brain show statistically significant differences (at $p<0.001, t$ test), comparing $0 \%$ vs $2 \%$ Chol diet.

$\|$ Values for serum and liver, but not retina or brain, show statistically significant differences (at $p<0.001, t$ test), comparing $0 \% v s 2 \%$ Chol diet. 
Serum sterol levels and composition also showed dramatic changes, comparing the $0 \%$ Chol versus $2 \%$ Chol groups (Table 1). The $7 \mathrm{DHC} / \mathrm{Chol}$ mole ratio in the $2 \%$ Chol group was only 0.10 , representing a 73 -fold drop compared with the $0 \%$ Chol group, largely due to the dramatic (32-fold) rise in Chol content to near-normal levels and the 2-fold drop in 7DHC level. Also, total serum sterols increased 4.5-fold to normal control levels $(115 \mathrm{mg} / \mathrm{dL})$ with Chol feeding. These observations paralleled those in liver, where the 7DHC/Chol ratio went from $5.52( \pm 2.68, n=6)$ in the $0 \%$ Chol group to $0.03( \pm 0.01, n=6)$ in the $2 \%$ Chol group (an 184-fold change), due to a 25 -fold increase in cholesterol and a 5.8 -fold decrease in 7DHC, resulting in a nearly 5-fold increase in total sterol content (mg/g wet weight of tissue). In contrast, no such dramatic shifts were observed in the sterol content or composition of brain upon dietary cholesterol supplementation. The 7DHC/Chol mole ratio in brain went from $2.95( \pm 1.78, n=$ 6 ) in the $0 \%$ Chol group to $1.95( \pm 1.35)$ in the $2 \%$ Chol group, but this apparent difference was not statistically significant, nor were there statistically significant alterations in brain 7DHC, Chol, or total sterol levels with dietary cholesterol supplementation.

Effect of dietary Chol supplementation on rod and cone function. Overall retinal function was examined using rod (Fig. 1) and cone (Fig. 2) ERG recordings. In comparison to age-matched control rats, ERG of animals administered AY9944 alone (0\% Chol group) exhibited dramatically reduced response amplitudes and prolonged implicit times, as previously reported (11). Dietary cholesterol supplementation produced relatively modest improvements in the dark-adapted
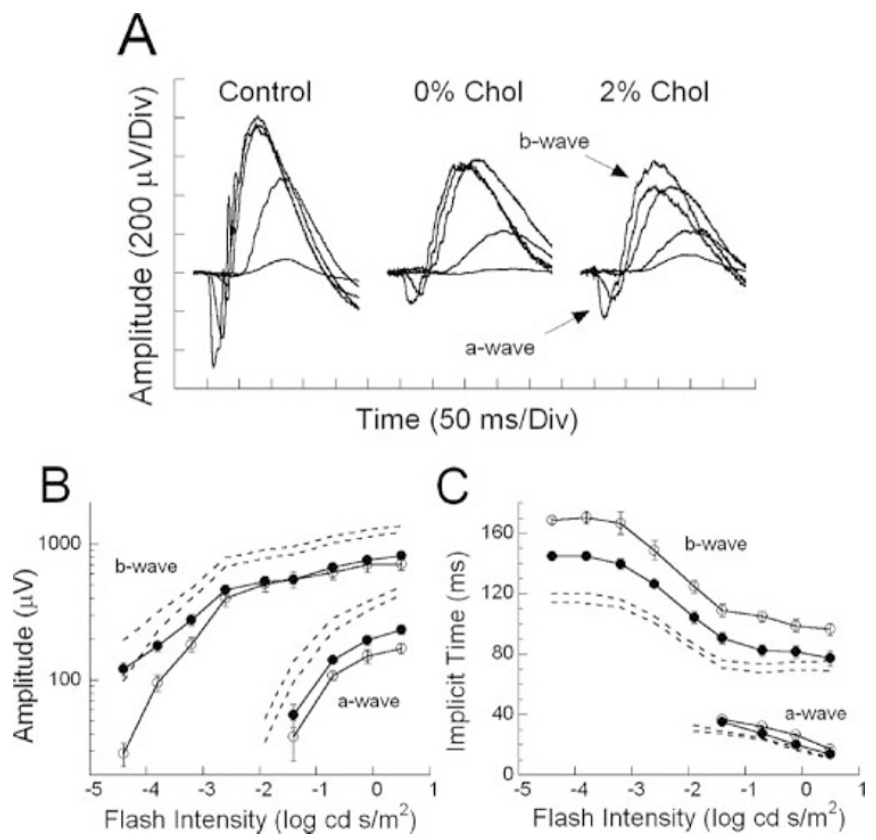

Figure 1. Dark-adapted electroretinograms. (A) Rod ERG from an agematched control rat (left) and rats treated with AY9944 on a Chol-free (middle) or a $2 \%$ cholesterol diet (right). A total of five responses obtained to strobe flash stimuli from -4.4 to $0.5 \log \mathrm{cd} \mathrm{s} / \mathrm{m}^{2}$ are overlain per analysis. Intensity-response functions for the amplitude $(B)$ and implicit times $(C)$ of the a- and b-wave components of the dark-adapted ERG are shown. Data represent mean values ( $\pm \mathrm{SEM}, n=6)$. $0 \%$ Chol $(\bigcirc) ; 2 \%$ Chol (O). Dashed lines indicate the $95 \%$ confidence interval for control rats.
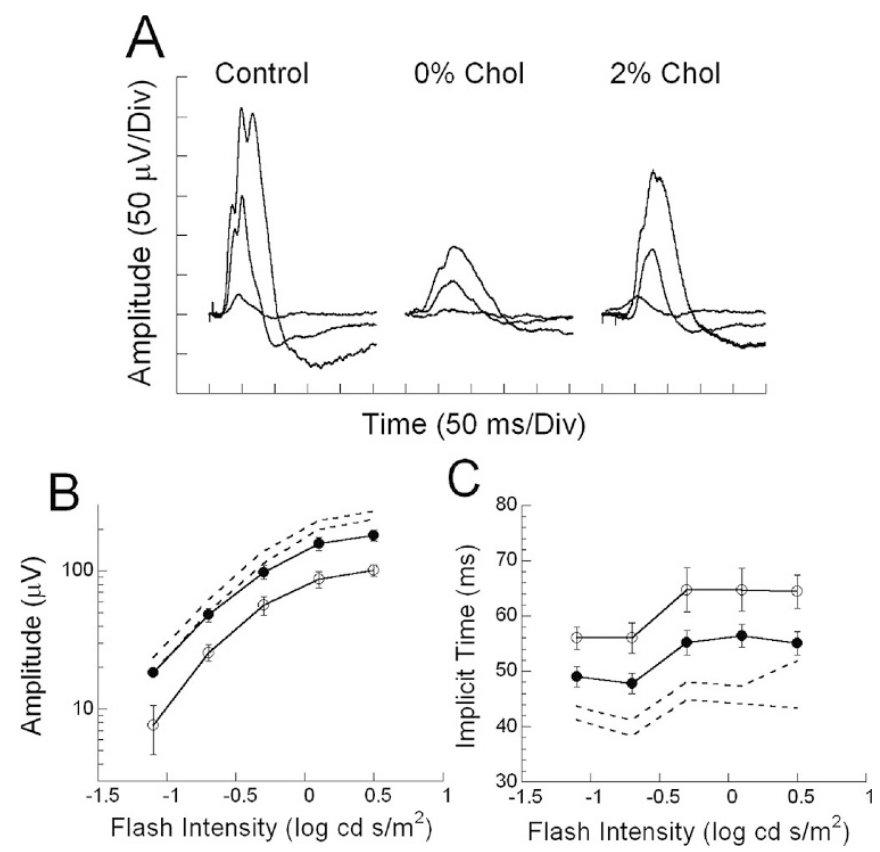

Figure 2. Light-adapted electroretinograms. (A) Cone ERG from an agematched control rat (left) and rats treated with AY9944 on a Chol-free (middle) or a $2 \%$ Chol diet (right). A total of five responses obtained to strobe flash stimuli from -1.1 to $0.5 \log \mathrm{cd} \mathrm{s} / \mathrm{m}^{2}$ are overlain per analysis. Intensityresponse functions for the amplitude $(B)$ or implicit time $(C)$ of the cone ERG are shown. Data points represent mean values ( \pm SEM, $n=6) .0 \% \mathrm{Chol}(\mathrm{O})$; $2 \%$ Chol (O). Dashed lines indicate the $95 \%$ confidence interval for control rats.

(rod) ERG a- or b-wave amplitudes. Across the intensity range where a-waves could be measured $\left(\geq-1.4 \log \mathrm{cd} \mathrm{s} / \mathrm{m}^{2}\right)$, a-wave amplitudes obtained from the $2 \%$ Chol group were $36 \pm 7 \%$ larger than those from the $0 \%$ Chol group $(p<$ 0.05 ). Across all stimulus conditions the amplitude of the b-wave was also larger in the $2 \%$ Chol than in the $0 \%$ Chol group, but this difference was not statistically significant. Moreover, this increase had a marked intensity dependence, where responses to low intensity stimuli were substantially larger in the $2 \%$ Chol group while responses to the five highest intensity stimuli were only $7 \%$ larger in the $2 \%$ Chol group (Fig. 1B). However, the delays in rod ERG a- and b-wave timing induced by AY9944 treatment (Fig. 1C) were significantly improved by cholesterol supplementation $(p<0.01)$. On average, a- and b-wave implicit times were decreased by $3.8 \pm 2 \mathrm{~ms}$ and $21.8 \pm 3.3 \mathrm{~ms}$, respectively, in the $2 \%$ Chol group. Nevertheless, both measures were above the normal range in the $2 \%$ Chol group. More dramatic improvements were observed in the light-adapted (cone) ERG following dietary Chol supplementation (Fig. 2). Across all stimulus conditions, cone ERG in the 2\% Chol group were $92 \pm 27 \%$ larger in amplitude $(p<0.05)$ and had implicit times $8.5 \pm$ $1.1 \mathrm{~ms}$ faster $(p<0.01)$ than the $0 \%$ Chol group, approaching, but not within, the normal range.

Effect of dietary Chol supplementation on retinal structure. Age-matched control rats exhibited normal retinal architecture; rats treated with AY9944 alone exhibited pathologic changes in the RPE, reduction in ROS length, and thinning of the ONL, as previously described (11). Figure 3 presents 


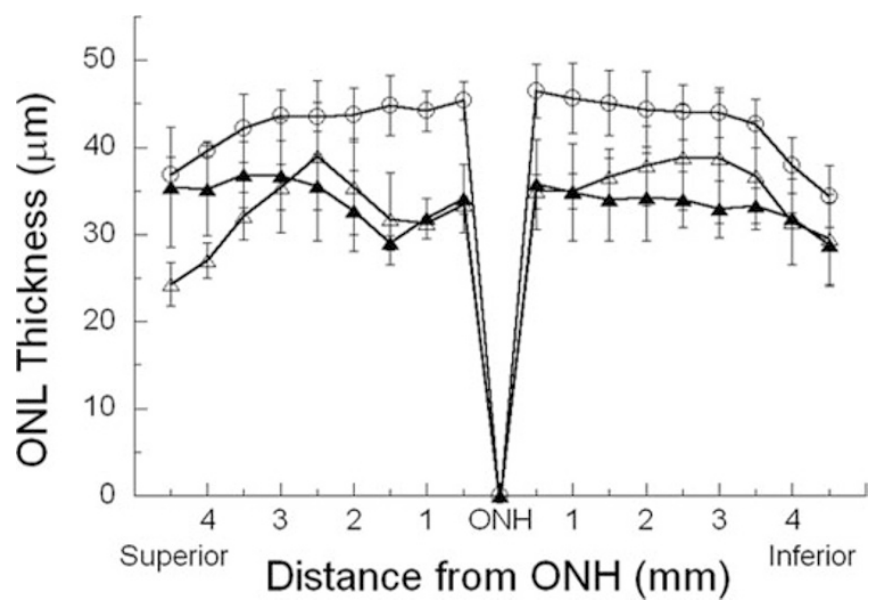

Figure 3. Quantitative morphometric analysis of rat retina ONL thickness. Data represent mean $( \pm \mathrm{SD})$ values taken at $0.5-\mathrm{mm}$ intervals from the optic nerve head $(\mathrm{ONH})$ along the vertical meridian (15 individual measurements per point, $n=4$ per condition). Control $(\bullet)$; AY9944 alone ( $\triangle$ ); AY9944 + $2 \% \operatorname{chol}(\mathbf{\Delta})$.

quantitative morphometric data regarding ONL thickness values (mean $\pm \mathrm{SD})$ as a function of distance from the optic nerve head $(\mathrm{ONH})$ along the vertical meridian, comparing AY9944-treated rats in the $0 \%$ Chol versus $2 \%$ Chol treatment groups and age-matched controls. This provides a quantitative, geographic index of photoreceptor survival: as photoreceptors degenerate and die, the ONL nuclei population diminishes with resultant thinning of the ONL. The mean ONL thickness of retinas in the 0\% Chol AY9944 treatment group $(33.9 \pm 4.2 \mu \mathrm{m})$ across the vertical meridian was approximately $26 \%$ less $(p<0.001)$ than that of control retinas $(42.7 \pm 3.3$ $\mu \mathrm{m})$, corresponding to an average loss of two vertical rows of photoreceptor nuclei in AY9944-treated rats, compared with controls. Dietary Chol supplementation afforded no significant sparing effect: mean ONL thickness $(33.8 \pm 2.3 \mu \mathrm{m})$ was reduced $21 \%(p<0.001)$, relative to controls, but there was no statistically significant difference between the two AY9944 treatment groups $(p>0.05)$. These data agree with previously obtained morphometric data comparing AY9944-treated versus control retinas (11) and are consistent with the darkadapted ERG data described above. Due to capricious retinal detachment in many of the eyes in both AY9944 treatment groups and suboptimal alignment of rods at several loci within the retinal sections, reliable quantitative morphometric analysis of ROS length was not possible. Qualitatively, however, ROS lengths in both AY9944 treatment groups (average 20 $\mu \mathrm{m})$ were appreciably different from ROS lengths in control retinas $(28 \mu \mathrm{m})$, but there were no obvious differences between the AY9944 treatment groups. These results are in good agreement with those we obtained previously (11), where 10 wk of AY9944 treatment caused a loss of about one-third in ROS length, relative to controls.

Figure 4 shows a quantitative comparison of ONL pyknotic nuclei counts at 1-mm intervals along the vertical meridian for retinas from all three groups. Few pyknotic nuclei were observed in the ONL of control animals (mean \pm SD values: $0.21 \pm 0.44$ per $\mathrm{mm}$ ). In contrast, animals treated with AY9944, whether given dietary cholesterol supplementation

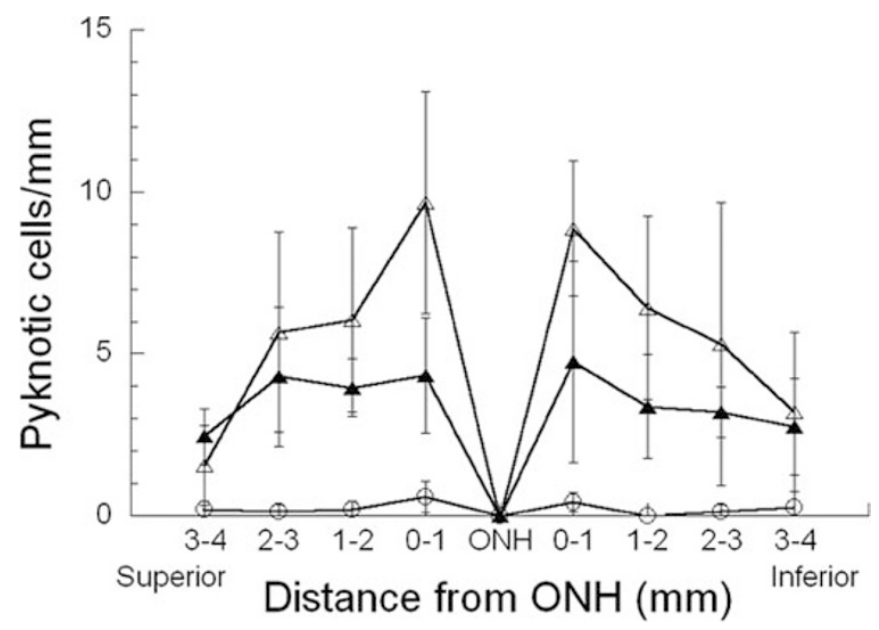

Figure 4. Effect of AY9944 treatment and Chol supplementation on ONL pyknosis. Morphometric data are mean values $( \pm \mathrm{SD})$ per 1-mm expanse along the vertical meridian (three or more individual measurements per point, $n=4$ per

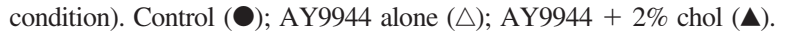

or not, had greater numbers of pyknotic nuclei in the ONL ( $>20$-fold higher). In the $0 \%$ Chol group there were $5.9 \pm 3.8$ pyknotic ONL nuclei per millimeter, whereas in the $2 \%$ Chol group there were $4.1 \pm 3.0$ pyknotic nuclei per millimeter. The mean number of pyknotic nuclei at almost every locus was lower in the $2 \%$ Chol group compared with the $0 \%$ Chol group (ca. 30\%), and this difference was statistically significant $(p<0.007)$. Hence, dietary Chol supplementation had a significant sparing effect on photoreceptor cell death, although not substantial enough to prevent the reduction in ONL thickness.

\section{DISCUSSION}

Herein, we demonstrate for the first time that dietary Chol supplementation can dramatically alter the steady-state sterol composition of the retina and provides concomitant partial rescue of visual function under conditions that mimic SLOS. Notably, these results are more dramatic than outcomes heretofore achieved with SLOS patients given Chol supplementation therapy (20-23). SLOS involves an inborn error in Chol biosynthesis resulting in accumulation of 7DHC and attenuation of Chol levels in all bodily tissues (1-3). This holds true for the AY9944-induced rat model of SLOS, where we previously reported such alterations in the sterol composition of the retina, with correlative histologic and electrophysiological abnormalities (11). The fact that a high-Chol diet can cause such marked changes in the steady-state sterol composition of the retina proves the blood-retina barrier is readily permeable to Chol, consistent with a recent study (25).

The permeability of the blood-retina barrier to Chol is in striking contrast to the blood-brain barrier, which excludes Chol (24). Our experimental data (Table 1) are fully consistent with this: no statistically significant change in the steady-state sterol content of rat brains was observed in response to a high-Chol diet under conditions where neural retina Chol content increased nearly 3 -fold, relative to rats on a Chol-free diet. The avid uptake of blood-borne Chol by the retina is encouraging with regard to the potential efficacy of dietary 
Chol supplementation for treating SLOS-associated retinopathy.

In our SLOS rat model, rod and cone ERG response amplitudes are markedly reduced, implicit times are prolonged, and rod sensitivities and maximum photoresponse are reduced nearly 2-fold (11), similar to human SLOS patients (16). Here we showed that dietary Chol supplementation modestly improved rod photoreceptor function in the SLOS rat model (Fig. 1), notably a-wave amplitudes and a- and b-wave implicit times. However, rod b-wave amplitudes were not significantly improved, except at low stimulus intensities, by Chol supplementation. This may reflect the dynamics of remodeling that occur at the synapse between rod photoreceptors (which generate the dark-adapted ERG a-wave) (26) and rod depolarizing bipolar cells (which generate the dark-adapted ERG b-wave) $(27,28)$ in response to photoreceptor degeneration $(29)$.

In contrast, a $2 \%$ Chol diet led to significant improvement in cone photoreceptor function in the SLOS rat model (Fig. 2): light-adapted ERG amplitudes were dramatically increased (nearly 2-fold) relative to AY9944-treated rats on Chol-free diet, and implicit times were shorter. When one considers these results within the context of human vision, the potential impact becomes more evident. While both rat and human retina photoreceptor populations are rod-dominant (ca. 2-3\% cones in rat retina, ca. 5-6\% cones in human retina), the human retina (unlike that of nonprimates) contains a geographically distinct, cone-rich macula (particularly the fovea, in the center of the macula), which affords high visual acuity. Hence, with the reasonable assumption that the fundamental cell biology of cones is similar in rats and humans, and given the observed improvement in cone function afforded by cholesterol supplementation in the SLOS rat model, one would predict that visual function improvements should occur in SLOS patients given cholesterol supplementation $(22,23)$. However, extrapolating results obtained with rats directly to humans, with regard to the amount of dietary Chol necessary to afford such visual improvement, is both difficult and illadvised. Also, why cone function is preferentially improved, in comparison with rods, by Chol supplementation in rats is unclear and will require additional studies.

Despite the marked trend toward normalization of retinal steady-state sterol composition and improvement of cone electrophysiology with dietary Chol supplementation in the SLOS rat model, photoreceptor degeneration occurs in animals treated with AY9944, regardless of diet, and all photoreceptor metrics were far from control values. This indicates that the chosen treatment regimen is not yet optimal. However, AY9944-treated rats raised on a $2 \%$ Chol diet exhibited fewer pyknotic photoreceptor nuclei than those raised on a $0 \%$ Chol diet. We believe this indicates that Chol abnormalities are not the sole significant factor promoting pathologic features in SLOS, particularly in the retina. The diagnostic biochemical hallmark of SLOS is the abnormal accumulation of 7DHC in bodily tissues (1-3). 7DHC is extraordinarily sensitive to auto-oxidation and photo-oxidation, forming oxysterols that are extremely toxic to cells (30). As detailed elsewhere $(18,19,31)$, the accumulation of $7 \mathrm{DHC}$ in the retina, with its high oxygen tension, would be expected to result in formation of such cytotoxic oxysterols, causing dysfunction and cell death in photoreceptors and other retinal cells. Furthermore, 7DHC may potentiate the generalized oxidation of unsaturated lipids, particularly polyunsaturated fatty acids (PUFA), to lipid peroxides. Photoreceptor outer segment membranes are highly enriched in PUFA (32). We have shown recently $(18,19)$ that: a) the SLOS rat model is more susceptible to intense light-induced retinal degeneration than normal albino rats; b) the histologic damage correlates with levels of lipid peroxides; c) ERG deficits and levels of lipid peroxides in retinas of SLOS rats under normal ambient lighting conditions are comparable to those in retinas of light-damaged (but otherwise normal) albino rats; and d) pretreatment of rats with a free radical scavenger (dimethylthiourea) affords substantial protection from light-induced retinal damage in both normal albino and SLOS rats. In fact, lipid peroxidation has been linked to retinal photodamage by other investigators (33-35). Hence, combining an antioxidant regimen with a high-Chol diet would seem appropriate to evaluate as an improved therapeutic intervention in SLOS patients.

\section{REFERENCES}

1. Kelley RI 2000 Inborn errors of cholesterol biosynthesis. Adv Pediatr 47:1-53

2. Porter FD 2002 Malformation syndromes due to inborn errors of cholesterol synthesis. J Clin Invest 110:715-724

3. Herman GE 2003 Disorders of cholesterol biosynthesis: prototypic metabolic malformation syndromes. Hum Mol Genet 12:R75-R88

4. Smith DW, Lemli L, Opitz JM 1964 A newly recognized syndrome of multiple congenital anomalies. J Pediatr 64:210-217

5. Irons M, Elias ER, Salen G, Tint GS, Batta AK 1993 Defective cholesterol biosynthesis in Smith-Lemli-Opitz syndrome. Lancet 341:1414

6. Tint GS, Irons M, Elias ER, Batta AK, Frieden R, Chen TS, Salen G 1994 Defective cholesterol biosynthesis associated with the Smith-Lemli-Opitz syndrome. N Engl J Med 330:107-113

7. Waterham HR, Wanders RJ 2000 Biochemical and genetic aspects of 7-dehydrocholesterol reductase and Smith-Lemli-Opitz syndrome. Biochim Biophys Acta 1529:340-356

8. Correa-Cerro LS, Porter FD 2005 3beta-hydroxysterol Delta7-reductase and the Smith-Lemli-Opitz syndrome. Mol Genet Metab 84:112-126

9. Yu H, Patel SB 2005 Recent insights into the Smith-Lemli-Opitz syndrome. Clin Genet 68:383-391

10. Battaile KP, Battaile BC, Merkens LS, Maslen GL, Steiner RD 2001 Carrier frequency of the common mutation IVS8-1G $>C$ in DHCR7 and estimate of the expected incidence of Smith-Lemli-Opitz syndrome. Mol Genet Metab 72:67-71

11. Fliesler SJ, Peachey NS, Richards MJ, Nagel BA, Vaughn DK 2004 Retinal degeneration in a rodent model of Smith-Lemli-Opitz syndrome. Arch Ophthalmol 122:1190-1200

12. Kolf-Clauw M, Chevy F, Wolf C, Siliart B, Citadelle D, Roux C 1996 Inhibition of 7-dehydrocholesterol reductase by the teratogen AY9944: a rat model for SmithLemli-Opitz syndrome. Teratology 54:115-125

13. Fliesler SJ, Richards MJ, Miller C-Y, Peachey NS 1999 Marked alteration of sterol metabolism and composition without compromising retinal development or function. Invest Ophthalmol Vis Sci 40:1792-1801

14. Dvornik D, Kraml M, Dubuc J, Givner M, Gaudry R 1963 A novel mode of inhibition of cholesterol biosynthesis. J Am Chem Soc 85:3309_

15. Givner ML, Dvornik D 1965 Agents affecting lipid metabolism-XV. Biochemical studies with the cholesterol biosynthesis inhibitor AY-9944 in young and mature rats. Biochem Pharmacol 14:611-619

16. Elias ER, Hansen RM, Irons M, Quinn NB, Fulton AB 2003 Rod photoreceptor responses in children with Smith-Lemli-Opitz syndrome. Arch Ophthalmol 121:1738-1743

17. Kretzer FL, Hittner HM, Mehta RS 1981 Ocular manifestations of the Smith-LemliOpitz syndrome. Arch Ophthalmol 99:2000-2006

18. Vaughan DK, Peachey NS, Richards MJ, Buchan B, Fliesler SJ 2006 Light-induced exacerbation of retinal degeneration in a rat model of Smith-Lemli-Opitz syndrome. Exp Eye Res 82:496-504

19. Richards MJ, Nagel BA, Fliesler SJ 2006 Lipid hydroperoxide formation in the retina: correlation with retinal degeneration and light damage in a rat model of Smith-Lemli-Opitz syndrome. Exp Eye Res 82:538-541 
20. Irons M, Elias ER, Abuelo D, Bull MJ, Greene CL, Johnson VP, Keppen L, Schanen C, Tint GS, Salen G 1997 Treatment of Smith-Lemli-Opitz syndrome: results of a multicenter trial. Am J Med Genet 68:311-314

21. Nwokoro NA, Mulvihill JJ 1997 Cholesterol and bile acid replacement therapy in children and adults with Smith-Lemli-Opitz (SLO/RSH) syndrome. Am J Med Genet 68:315-321

22. Linck LM, Lin DS, Flavell D, Connor WE, Steiner RD 2000 Cholesterol supplementation with egg yolk increases plasma cholesterol and decreases plasma 7-dehydrocholesterol in Smith-Lemli-Opitz syndrome. Am J Med Genet 93:360-365

23. Sikora DM, Ruggiero M, Petit-Kekel K, Merkens LS, Connor WE, Steiner RD 2004 Cholesterol supplementation does not improve developmental progress in SmithLemli-Opitz syndrome. J Pediatr 144:783-791

24. Bjorkhem I, Meaney S 2004 Brain cholesterol: long secret life behind a barrier. Arterioscler Thromb Vasc Biol 24:806-815

25. Tserentsoodol N, Sztein J, Campos M, Gordiyenko N, Farris RN, Lee JW, Fliesler SJ, Rodriguez IR 2006 Uptake of cholesterol by the retina occurs primarily via an LDL receptor-mediated process. Mol Vis 12:1306-1318

26. Lamb TD 1996 Transduction in human photoreceptors. Aust N Z J Ophthalmol 24:105-110

27. Kofuji P, Ceelen P, Zahs KR, Surbeck LW, Lester HA, Newman EA 2000 Genetic inactivation of an inwardly rectifying potassium channel (Kir4.1 subunit) in mice: phenotypic impact in retina. J Neurosci 20:5733-5740
28. Robson JG, Frishman LJ 1995 Photoreceptor and bipolar cell contributions to the cat electroretinogram: a kinetic model for the early part of the flash response. J Opt Soc Am A Opt Image Sci Vis 13:613-622

29. Aleman TS, LaVail MM, Montemayor R, Ying G, Maguire MM, Laties AM, Jacobson SG, Cideciyan AV 2001 Augmented rod bipolar cell function in partial receptor loss: an ERG study in $\mathrm{P} 23 \mathrm{H}$ rhodopsin transgenic and aging normal rats. Vision Res 41:2779-2797

30. Girotti AW. 2002 Cholesterol-derived hydroperoxides: generation and reactivity in biological systems. In: Fliesler SJ (ed) Sterols and Oxysterols: Chemistry, Biology and Pathobiology. Research Signpost, Kerala, India, pp 121-139

31. Fliesler SJ. 2002 Effects of cholesterol biosynthesis inhibitors on retinal development, structure, and function. In: Fliesler SJ (ed) Sterols and Oxysterols: Chemistry, Biology and Pathobiology. Research Signpost, Kerala, India, pp 77-109

32. Fliesler SJ, Anderson RE 1983 Chemistry and metabolism of lipids in the vertebrate retina. Prog Lipid Res 22:79-131

33. Organisciak DT, Winkler BS 1994 Retinal light damage: practical and theoretica considerations. Prog Ret Eye Res 13:1-29

34. Boulton M, Rozanowska M, Rozanowski B 2001 Retinal photodamage. J Photochem Photobiol B 64:144-161

35. Wenzel A, Grimm C, Samardzija M, Reme CE 2005 Molecular mechanisms of light-induced photoreceptor apoptosis and neuroprotection for retinal degeneration Prog Retin Eye Res 24:275-306 